\title{
Compact high-temperature cell for Brillouin scattering measurements
}

\author{
Stanislav V. Sinogeikin, ${ }^{\text {a) }}$ Jennifer M. Jackson, ${ }^{\text {b) }}$ and Bridget O’Neill \\ Department of Geology, University of Illinois at Urbana-Champaign, Urbana, Illinois 61801 \\ James W. Palko \\ Department of Materials Science and Engineering, University of Illinois at Urbana-Champaign, Urbana, \\ Illinois 61801 \\ Jay D. Bass \\ Department of Geology, University of Illinois at Urbana-Champaign, Urbana, Illinois 61801
}

(Received 20 July 1999; accepted for publication 27 September 1999)

\begin{abstract}
A compact ceramic high-temperature cell for Brillouin spectroscopy was designed and tested. The cell can be mounted onto a three- or four-circle goniometer and allows collection of the full set of elastic constants of minerals to temperatures in excess of $1500 \mathrm{~K}$ from samples with dimensions of $100 \times 100 \times 20 \mu \mathrm{m}$ or smaller. As a test of the instrument the single-crystal elastic constants of $\mathrm{MgO}$ were measured to $1510(10) \mathrm{K}$, and are found to be in excellent agreement with earlier independent results. The high-temperature cell should be useful for other types of spectroscopic measurements, and is especially useful in situations where spectral properties vary with the scattering geometry.

(C) 2000 American Institute of Physics. [S0034-6748(00)02201-2]
\end{abstract}

\section{INTRODUCTION}

A knowledge of elastic and other fundamental properties of materials at high temperature is important from both the technological and scientific points of view. High-temperature elasticity data provide fundamental information on the interatomic forces in solids. They are important in geophysics for constructing reliable mineralogical models of the Earth's interior by comparing laboratory elasticity data on minerals with seismologically determined properties. For geophysical purposes and for materials that may be used in hightemperature applications, it is obviously advantageous to characterize materials at elevated temperatures.

Brillouin scattering provides a means of studying the high-temperature elasticity of materials. Being a purely optical method, it does not require any mechanical contact between the sample and measuring equipment, and can be performed on very small samples with dimensions comparable to the size of the focused beam. In addition, Brillouin spectroscopy can be used to study other properties at high temperature such as the refractive index, ${ }^{1,2}$ photoelastic properties, ${ }^{3}$ hypersonic attenuation, ${ }^{4}$ and the mechanisms of phase transitions. ${ }^{2,4-9}$

Despite the considerable possibilities described above, there have been relatively few high-temperature Brillouin spectroscopy experiments reported in the literature. Most have typically used bulky furnaces with limited optical access and restricted possibilities for data collection, and the crystal samples typically exceeded several millimeters in each dimension. Although this does not present a problem for work with common materials, some materials of interest cannot be synthesized with crystal sizes more than a few

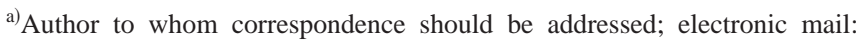
sinogeik@uiuc.edu

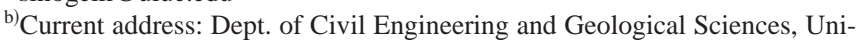
versity of Notre Dame, Notre Dame, IN 46556.
}

hundreds of microns. Other limitations may include (1) difficulty in the precise orientation of samples, (2) very limited angular access to the sample, which restricts the number of scattering directions and elastic constants that can be obtained, especially for symmetries lower than cubic, and (3) difficulty in measuring the refractive index as a function of temperature independently.

To overcome these problems we designed a ceramic high-temperature cell for single-crystal Brillouin spectroscopy to temperatures in excess of $1000{ }^{\circ} \mathrm{C}$. The cell is compact (about $5 \mathrm{~cm}$ in the maximum dimension), and easily fits onto any standard three- or four-circle goniometer, allowing a number of additional applications such as x-ray scattering, etc. Our Brillouin cell utilizes a symmetric scattering geometry ${ }^{11}$ which yields velocity measurements independent of the refractive index. This is important because the refractive index can change appreciably with temperature. Because the cell mounts onto a goniometer, one obtains very good control of the scattering geometry, and any phonon direction within a plane of the platelet can be easily sampled by changing the $\chi$-angle setting of the goniometer. As a result, the complete set of elastic constants as a function of temperature for crystals with symmetry higher than monoclinic can be obtained from a single sample if the sample plane intersects all three crystallographic axes. ${ }^{12}$ Finally, the cell allows us to work with crystals approximately $100 \times 100 \times 20 \mu \mathrm{m}$ or smaller. ${ }^{13}$ It should be straightforward to modify the cell for experiments in a controlled atmosphere.

\section{CELL DESCRIPTION}

The geometrical design of the high-temperature Brillouin cell is similar to that of a large-opening Merill-Bassett diamond anvil cell ${ }^{14}$ (Fig. 1). However the cell described here is designed to generate high temperature only, not pres- 


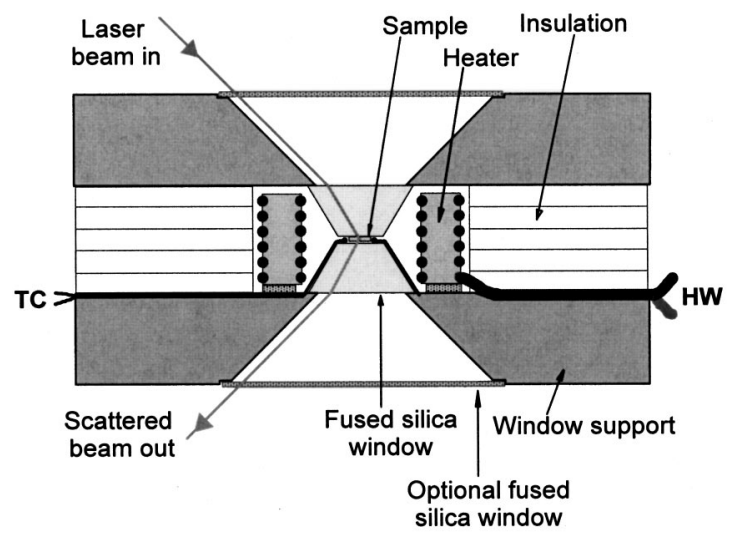

FIG. 1. Schematic diagram of Brillouin scattering in the high-temperature Brillouin cell. TC —-thermocouples; HW—heating wires.

sure. The cell consists of five major components: the cell base, window supports, windows, heater, and thermocouples (Fig. 2), each of which is described below.

The main body of the cell is constructed from machinable alumina silicate ceramic, which is inexpensive and is easily obtained from a variety of manufacturers and distributors (e.g., McMaster-Carr Supply Company). This type of ceramic is rated to withstand temperatures of up to $1370 \mathrm{~K}$, and has proved reliable for sample temperatures well in excess of $1500 \mathrm{~K}$. For higher temperatures, a different machinable ceramic can be readily substituted (e.g., RESCOR 960, Cotronics). Although RESCOR 960 ceramic is more expensive, it has better thermal shock and mechanical properties at higher temperatures than alumina silicate ceramic.

The advantage of using a machinable ceramic is that it is soft and easily shaped with conventional tools. Since these types of ceramics are usually quite brittle, care must be taken not to chip sharp edges during machining. After the parts are machined, they are heat treated according to the manufacturer's instructions to obtain their maximum strength and thermal stability. Note that during heat treatment the alumina silicate ceramic can expand slightly ( $1.8 \%$ in our case), which must be taken into account when fitting with metal parts such as the goniometer socket. A number of machinable ceramics (e.g., RESCOR 960) do not require heat treat-

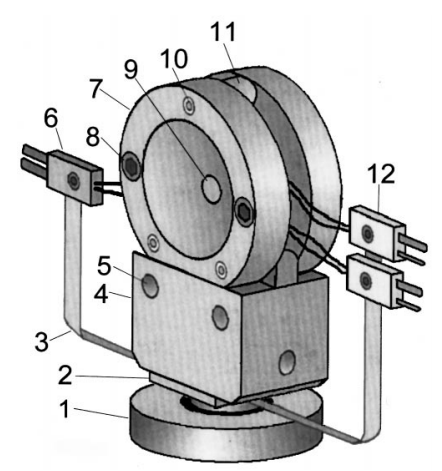

FIG. 2. View of the entire high-temperature cell. 1-Goniometer socket; 2-insulating ceramic plate; 3-metal support for the thermocouple and heater connectors; 4 - ceramic base; 5-threaded holes for water cooling; 6-heater connector; 7-ceramic window support; 8-screws; 9-fused silica window; 10—ceramic guide pins; 11 -ceramic spacer; 12thermocouple connectors.

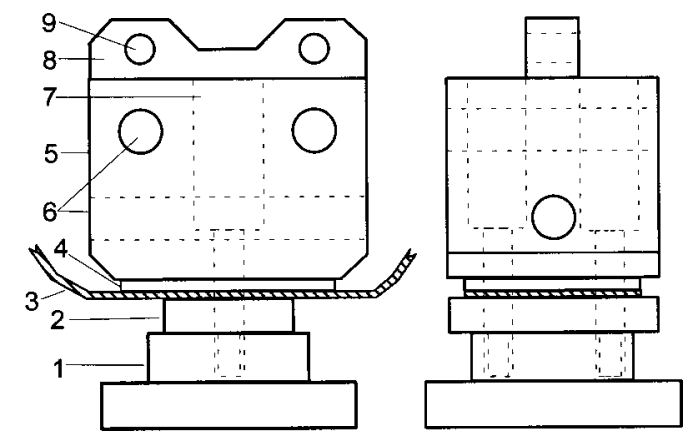

FIG. 3. Base of the high-temperature cell. 1-Goniometer socket; 2-ceramic plate; 3 -metal support for the heater and thermocouple connectors; 4-insulating ceramic fiber strip; 5-ceramic base; 6-threaded holes for optional water cooling; 7-screw well for connecting the ceramic base with the metal base; 8-spacer for mounting window supports; 9-holes for ceramic guide pins.

ment and can be used for a more precise match of cell components.

\section{A. Base}

The base of the cell (Fig. 3) consists of two main parts: a goniometer socket and a ceramic base. The goniometer socket was machined from brass. Alternatively, commercial sockets/height adapters can be obtained from any $\mathrm{x}$-ray equipment supplier.

The upper part of the cell base, to which the main body of the cell is mounted, was made from machinable alumina silicate ceramic. The spacer for mounting window supports in the upper part of the base is machined with a thickness which is slightly greater than the thickness of the two windows to allow for the thickness of the sample and the thermocouples. We used 1/8 in. $(3.175 \mathrm{~mm})$ thick windows and a thickness for the spacer bar of $6.5 \mathrm{~mm}$ to ensure a $0.15 \mathrm{~mm}$ gap between the windows.

Since the cell is mounted on a goniometer, special care should be taken to prevent the goniometer from overheating. The cell can be water cooled to minimize the temperature at the base. Due to the low thermal conductivity of the ceramic and holes for water cooling (which decrease the effective area for heat transfer), the base of the cell remained relatively cool $\left(60-70^{\circ} \mathrm{C}\right)$ without water cooling even at the highest temperature achieved $(1510 \mathrm{~K})$.

A strip of $1 \mathrm{~mm}$ thick stainless steel was placed between the lower and upper parts of the base and bent appropriately so it could provide support for electrical connectors (Fig. 2). A piece of insulating ceramic fiber strip was placed in between the connector support and the ceramic base. The goniometer socket and ceramic base were connected with two screws.

\section{B. Window supports}

Top and side views of the window support plates are shown in Fig. 4. The window supports were machined from 1.5 in. diam rods of machinable alumina silicate ceramic. The cone has an overall opening of $100^{\circ}$, which allows its use in a symmetric scattering geometry with up to a $90^{\circ}-95^{\circ}$ scattering angle. Since no pressure is applied to the cell, the 


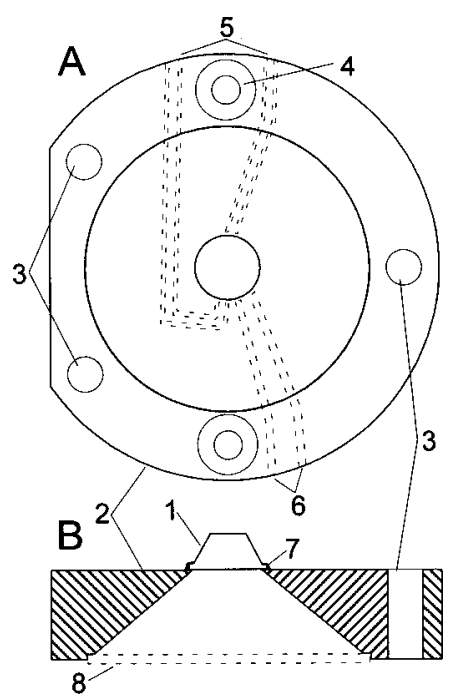

FIG. 4. Top view and cross section of the ceramic window support plate with the attached window. 1-Window; 2 -ceramic window support plate; 3-holes for guide pins; 4-screw hole; 5-grooves for thermocouple wires; 6-grooves for heater wires; 7-high-temperature cement; 8 - optional outer window.

diameter of the central hole in the supporting plate can be nearly as large as that of the windows.

The parallellness of the window supports is controlled by proper dimensions of ceramic spacers (one bar of the base and one tube on top sitting on guide pins). The distance between window supports can be increased by putting additional washers on top of the ceramic spacers.

The supporting plates have five through holes: three for ceramic guide pins and two for screws. The guide pins (standard ceramic thermocouple insulators) ensure proper positioning of the windows and connect the window supports with the base. In one of the two support plates the screw holes are threaded.

Before heat treatment we made grooves 1-1.5 mm deep which are used to recess and secure the thermocouple and heating wires (Fig. 4).

On the outer side of the window supports we cut 1-2 $\mathrm{mm}$ deep steps to allow gluing of additional silica-glass windows, which eliminate contact of the main windows with air and reduce the temperature gradient inside the cell. The trade-off is that the outside windows introduce additional astigmatism and reflect an appreciable part of the incident and scattered beams, diminishing the quality of the Brillouin spectra.

\section{Windows}

Brillouin spectra are geometry sensitive, and the scattering angle must be carefully controlled in any experiment. To ensure that no part of the incident or scattered beams is cut off, the ratio of window height to outside window diameter should not exceed a critical value which is defined by the refractive index of the window material used, the scattering geometry, and the numerical aperture of the focusing and collecting lenses. The maximum height to width ratio for the windows is easily calculated through Snell's law. For example, in our experiments we used windows made of fused silica. At a wavelength of $\lambda=5000 \AA$, the room temperature refractive index of the fused silica is about 1.46 and does not change appreciably with temperature. For a window thickness of $1 / 8 \mathrm{in} .(3.175 \mathrm{~mm})$ the diameter of the outside surface of the window should not be smaller than $4.8 \mathrm{~mm}$ in order to use an $80^{\circ}$ symmetric scattering geometry with $f$ $=3.3$ lenses and achieve a $1 \mathrm{~mm}$ working area inside the cell. Failure to meet these conditions will result in vignetting (shadowing part of the incident and/or scattered cones of light) and systematically lower the observed Brillouin shifts. A discussion of vignetting effects on Brillouin spectra is beyond the scope of this article; we refer the reader to the discussion by Oliver et al. ${ }^{15}$ who considered an optical geometry identical to ours in the context of Brillouin scattering with a diamond anvil cell. We note only that the dimensions of our high-temperature cell are easily altered to suit almost any optical configuration without significantly changing the performance of the cell.

The windows were machined from $1 / 8$ in. $(3.175 \mathrm{~mm})$ thick disks of fused silica with polished parallel faces. For windows $6 \mathrm{~mm}$ in diameter the disks were cut into 6 $\times 6 \mathrm{~mm}$ square or octagonal blocks. These blocks were glued with superglue to $6 \mathrm{~mm}$ diam silica-glass rods (with the polished surfaces of the blocks perpendicular to the axis of the rod). These rods were secured in a drill press, and the blocks were ground to the desired conical shape with a conventional diamond file.

The fused silica windows proved adequate to temperatures of up to $1500 \mathrm{~K}$, but can lose optical quality or soften at higher temperatures. The silica may also react with certain materials at high temperature. Alternative materials used for windows were sapphire and cubic zirconia. The advantage of these two materials are higher temperature stability, lower reactivity, and higher refractive indexes. This reduces the minimum window diameter-to-height ratio, which will result in a more uniform temperature distribution, and allows work with larger sample areas. The disadvantages are higher strength (which makes the machining of windows more difficult), high acoustic velocities and velocity anisotropy (which could complicate the Brillouin spectra of very thin samples), and higher astigmatism due to the higher refractive indexes.

Sapphire windows were also machined from $1 / 8$ in. thick polished sapphire disks. The windows, shaped as truncated octagonal pyramids, were cut using a fine diamond saw. $\mathrm{Cu}-$ bic zirconia windows were made from round diamond-cut jewelry obtained from a commercial gemstone dealer. A flat inner facet was hand ground and polished parallel to the table of the gemstone using a metal jig and a series of silicon carbide abrasive films $(30,5,1 \mu \mathrm{m}$ grit size). Excess material on the sides was ground off with silicon carbide sandpaper. Alternatively, the windows from either of the above materials can be easily machined using a lapidary faceting machine.

Note that only two parallel facets of the windows should have near-optical quality. The sides of the windows should be coarse ground, which makes it easier to glue the windows to window supports and to glue thermocouples to the windows.

The windows were attached to the window supports with 
either Cotronics 903 HPRT alumina adhesive or Cotronics 906 magnesia adhesive. We found that, since the alumina adhesive contains much finer powder than the magnesia adhesive, it is easier to work with, especially where very fine work is required. Nevertheless the magnesia adhesive shows less reactivity and better stability at high temperature.

\section{Heater}

The core of the heater was machined from a $1 / 2$ in. diam rod of alumina silicate ceramic. The rod was threaded inside and outside with a $1 / 4$ and $1 / 2$ in. $\times 40$ tpi tap and die, respectively, cut into $5 \mathrm{~mm}$ thick disks, and heat treated. A high ratio of the height to the inner diameter of the heater is very important for creating stable temperature conditions and maintaining small temperature gradients.

It was found that the double-coil design of the heater (the heating wire wound inside and outside of the heater core) produces more stable temperatures and smaller temperature gradients in the sample chamber. It also reduces the temperature of the heater wires needed to maintain a given temperature in the sample chamber, which allows higher experimental temperatures to be obtained. It was found beneficial to use different heating wires for the internal and external coils with higher power density for the internal coil. We used 0.01 in. Pt-30Rh wire for the internal coil and 0.005 in. $\mathrm{Pt}$ or $\mathrm{Pt}-30 \mathrm{Rh}$ for the external coil. Outside the heater, the two coils were connected together in parallel and lead to the high-temperature ceramic connector outside the cell.

The heater was powered with a digital dc power supply rated to a maximum of $18 \mathrm{~V}$ and $10 \mathrm{~A}$. The temperature was controlled to $0.5^{\circ}$ by controlling the output voltage. At any temperature the voltage did not exceed $14.6 \mathrm{~V}$ with a corresponding current of 6.6 A. At the highest temperature (1510 K) the output power was $96.6 \mathrm{~W}$.

To further increase the effectiveness of the heater, we packed all empty space in between the window supports with insulating ceramic fiber tape. This additional insulation decreases both power consumption and temperature gradients inside the cell.

\section{E. Temperature control}

The temperature was measured directly by two thermocouples fixed with high-temperature cement to the window. The cement also served to insulate the thermocouple wires from heating and conducting heat to the thermocouple (TC) junctions. The thermocouples were placed as close to the sample and as far away from the heater as possible.

We used type $K$ (Chromel-Alumel) thermocouples made of 0.005 in. diam wire. At temperatures above $1000^{\circ} \mathrm{C}$, the thermocouple wires oxidize and reliability can be lost after long durations at high temperatures (although covering them with an appropriate cement inhibits the oxidation). Therefore, at temperatures in excess of $1000^{\circ} \mathrm{C}$, Pt-based thermocouples (types $B, S, R$ ) are prefered, even though the sensitivity and reliability of type $K$ thermocouples at lower temperatures are superior.

The temperature in the cell is stable over time. Even at the highest temperatures, after thermal equilibration the tem-

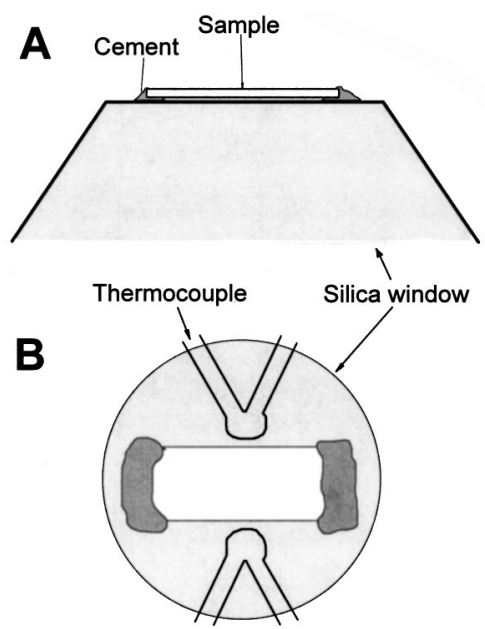

FIG. 5. Sample mounting on a window. The thermocouples shown are normally attached to an opposite window.

perature fluctuations did not exceed $2^{\circ}$ over several hours of operation. Since the cell is made from materials with very low thermal conductivity, the thermal gradient inside the cell (between thermocouples) is small. The thermal gradient is not perfectly symmetric. Preliminary measurements in the direction of the maximum thermal gradient show that this gradient does not exceed $10 \% \mathrm{~mm}$ at temperatures below $1000 \mathrm{~K}$ and $20 \% \mathrm{~mm}$ at temperatures around $1500 \mathrm{~K}$. The thermal gradient is very sensitive to the choice of materials for the cell and the configuration of the heater and windows.

\section{ELASTICITY OF MgO TO $1500 \mathrm{~K}$}

$\mathrm{MgO}$ is an ideal substance for testing our new furnace. It is cubic in structure, and therefore its elasticity is characterized by only three independent elastic constants. The hightemperature single-crystal elasticity of $\mathrm{MgO}$ has been measured by a number of different experimental techniques up to its melting point. The experimental measurements include ultrasonic (rectangular parallelepiped resonance) studies ${ }^{16,17}$ and Brillouin measurements. ${ }^{18}$ In addition, $\mathrm{MgO}$ is a very good Brillouin scatterer, large flawless single crystals can be easily obtained, and perfect cleavage along $\{100\}$ planes makes the orientation of samples easy. $\mathrm{MgO}$ is nonreactive, and the sample does not deteriorate in air even at temperatures exceeding $1500 \mathrm{~K}$.

The single-crystal elasticity measurements were performed on two samples of $\mathrm{MgO}$ by Brillouin scattering in two separate runs up to a temperature of $1510(10) \mathrm{K}$.

The slab of a synthetic single crystal of $\mathrm{MgO}$ was polished on both sides along the (100) direction to a thickness of $\approx 100 \mu \mathrm{m}$ and cleaved into plates of $\sim 1 \times 2 \mathrm{~mm}$. These plates were glued to the fused silica window with Cotronics high-temperature alumina or magnesia adhesive. Because of strong capillary forces some of the adhesive flowed under the sample and therefore lifted it from the surface of the window by $20-30 \mu \mathrm{m}$ (Fig. 5). Subsequent optical goniometry indicated that the angle between the face normals of the window and sample faces did not exceed $1^{\circ}$.

In both runs we utilized $80^{\circ}$ symmetric platelet scattering geometry with an $\operatorname{Ar}$-ion laser $(\lambda=5145 \AA)$ light source 


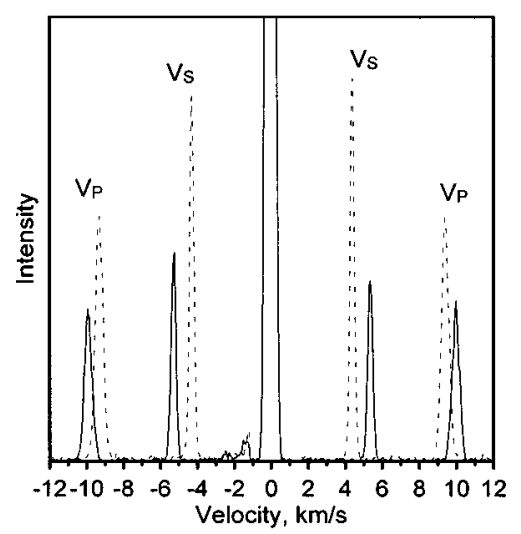

FIG. 6. Brillouin spectra of $\mathrm{MgO}$ in the [011] direction. Solid lines-Room temperature spectra; dashed lines-spectra at $1510(10) \mathrm{K}$. The intensities of the peaks are scaled to fit on the same plot. At $1510 \mathrm{~K}$, the actual intensity of the Brillouin peaks was a factor of 5 higher than at room temperature. Note the low background, even at $1510 \mathrm{~K}$; this indicates that thermal emission was effectively filtered out.

and a six-pass tandem Fabry-Pérot interferometer. Further description of the Brillouin system is given elsewhere. ${ }^{19}$ It is worth noting that the scattered light is filtered with a combination of a dispersing prism and a pinhole. This filters out all light except for the green region. Thus, thermal radiation does not show as a high background in Brillouin spectra, a typical example of which is shown in Fig. 6.

In the first run, alumina adhesive was used to attach the sample and thermocouples to the cell window. Data were obtained at temperatures of 295, 523, 773, and $1023 \mathrm{~K}$ (shown by circles in Figs. 7 and 8). Above 1050 K, one of the thermocouples broke. In addition, the optical quality of the $\mathrm{MgO}$ sample started to deteriorate, probably due to vapor reaction with the alumina adhesive, and the run was stopped. We found that the Chromel thermocouple wires had reacted with the alumina adhesive, and one of them was completely destroyed during the run. The alumina adhesive also reacted

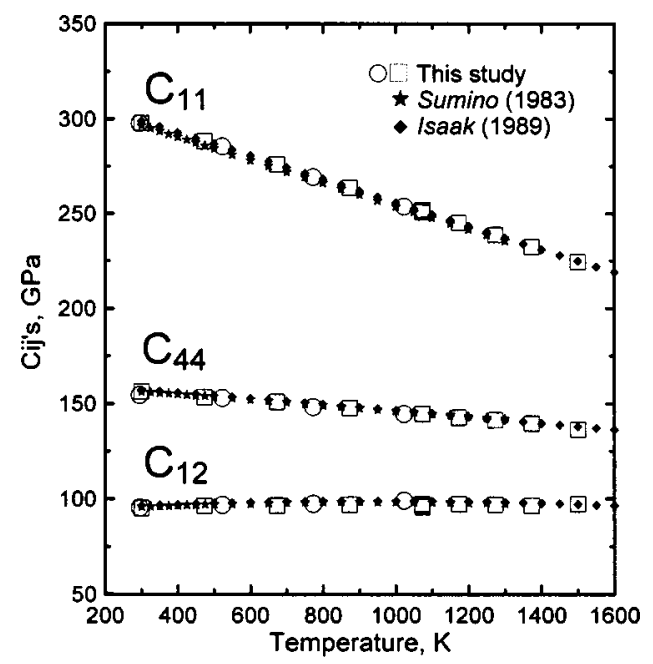

FIG. 7. Single-crystal elastic constants of $\mathrm{MgO}$ as a function of temperature. Open symbols (circles and squares) represent two different runs from this study. Closed symbols show ultrasonic data of Sumino et al. (Ref. 16) and of Isaak et al. (Ref. 17) for comparison. The size of the symbols is bigger than the experimental uncertainty.

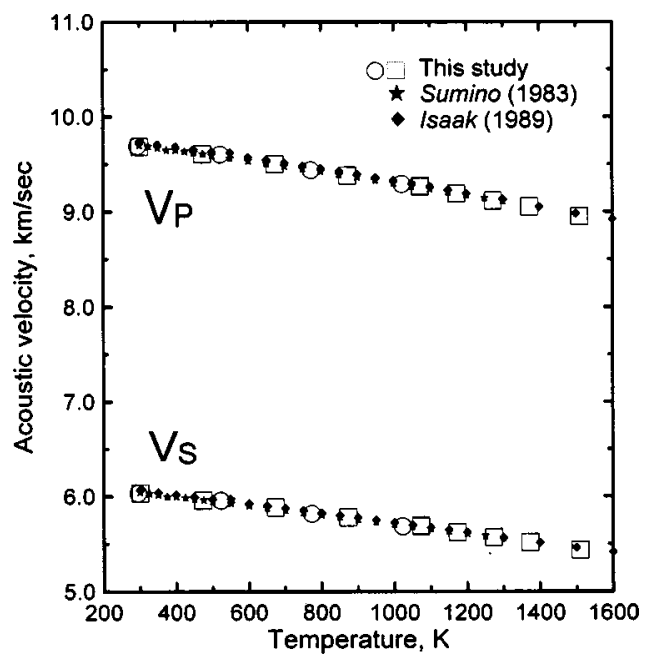

FIG. 8. Aggregate acoustic velocities in $\mathrm{MgO}$ as a function of temperature. The symbols have the same meaning as those in Fig. 7.

with the silica windows at high temperature, although the reaction was restricted to the contact zone only.

In the next run, we used magnesia adhesive to glue the thermocouples and sample to the window, and no reaction was observed to the maximum temperature of $1510 \mathrm{~K}$. The data were collected at temperature of $300,473,673,873$, $1073,1173,1273,1373$, and $1510 \mathrm{~K}$ (shown by squares in Figs. 7 and 8). After the data collection at 1273 and $1510 \mathrm{~K}$ the temperature was returned to $1073 \mathrm{~K}$ to check if there was any difference in velocity measurements made on increasing and decreasing temperatures. No systematic difference was observed, and both elastic moduli and aggregate acoustic velocities were in mutual agreement.

At temperatures of 295(1), 1073(5), and 1510(10) K the data were collected in more than 10 crystallographic directions (Fig. 9) which allowed us to solve for both orientation and single-crystal elastic moduli. ${ }^{12}$ Our results show that the calculated phonon directions at the three different tempera-

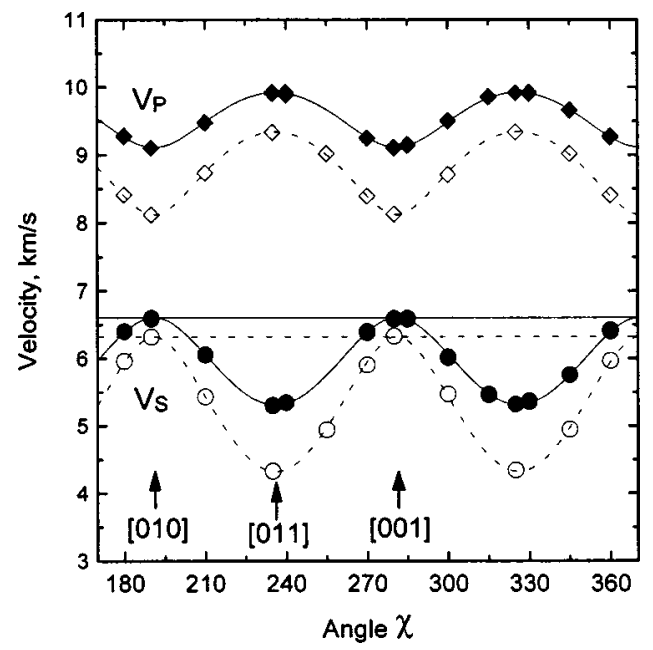

FIG. 9. Acoustic velocities in $\mathrm{MgO}$ as a function of crystallographic direction ( $\chi$ angle) at room temperature (closed symbols) and at $1510 \mathrm{~K}$ (open symbols). Acoustic velocities calculated from the best-fit elastic moduli at room temperature (solid line) and at $1510 \mathrm{~K}$ (dashed line). The [010], [011], and [001] crystallographic directions are marked. 
TABLE I. Single-crystal elastic constants, aggregate elastic moduli (VRH average), and acoustic velocities of $\mathrm{MgO}$ as a function of temperature. (The uncertainty in density is less than $0.1 \%$. The uncertainty in the elastic moduli is less than $1 \%$, and in the acoustic velocities it is less than $0.5 \%$.)

\begin{tabular}{|c|c|c|c|c|c|c|c|c|c|}
\hline $\begin{array}{l}\text { Run } \\
\text { No. }\end{array}$ & $\begin{array}{l}\text { Temperature } \\
\text { (K) }\end{array}$ & $\begin{array}{l}\text { Density } \\
(\mathrm{g} / \mathrm{cc})\end{array}$ & $\begin{array}{c}C_{11} \\
(\mathrm{GPa})\end{array}$ & $\begin{array}{c}C_{44} \\
(\mathrm{GPa})\end{array}$ & $\begin{array}{c}C_{12} \\
(\mathrm{GPa})\end{array}$ & $\begin{array}{c}K_{S} \\
(\mathrm{GPa})\end{array}$ & $\begin{array}{l}G_{\mathrm{VRH}} \\
(\mathrm{GPa})\end{array}$ & $\begin{array}{c}V_{P} \\
(\mathrm{~km} / \mathrm{s})\end{array}$ & $\begin{array}{c}V_{S} \\
(\mathrm{~km} / \mathrm{s})\end{array}$ \\
\hline $\operatorname{mg} 1 \mathrm{t} 1$ & $295(1)$ & 3.584 & 297.7 & 154.5 & 95.3 & 162.8 & 130.4 & 9.691 & 6.032 \\
\hline $\mathrm{mg} 1 \mathrm{t} 2$ & 523 & 3.555 & 285.5 & 152.9 & 96.7 & 159.6 & 126.0 & 9.600 & 5.953 \\
\hline $\operatorname{mg} 1 \mathrm{t} 3$ & $773(5)$ & 3.519 & 269.2 & 148.1 & 97.3 & 154.6 & 119.0 & 9.435 & 5.815 \\
\hline $\operatorname{mg} 1 \mathrm{t} 4$ & $1023(20)$ & 3.481 & 253.7 & 144.5 & 99.0 & 149.0 & 112.0 & 9.289 & 5.681 \\
\hline $\mathrm{mtf} 1$ & $300(2)$ & 3.584 & 297.8 & 156.4 & 95.2 & 162.7 & 131.4 & 9.710 & 6.055 \\
\hline $\mathrm{mtf} 2$ & 473 & 3.562 & 288.2 & 153.5 & 96.4 & 160.3 & 127.1 & 9.622 & 5.973 \\
\hline $\mathrm{mtf} 3$ & 673 & 3.534 & 276.1 & 151.0 & 96.6 & 156.4 & 122.6 & 9.513 & 5.888 \\
\hline $\mathrm{mtf} 4$ & 873 & 3.504 & 263.6 & 147.7 & 97.0 & 152.5 & 117.4 & 9.391 & 5.787 \\
\hline $\mathrm{mtf5}$ & 1073(5) & 3.474 & 251.7 & 144.6 & 97.2 & 148.7 & 112.4 & 9.271 & 5.688 \\
\hline mtf6 & 1173 & 3.458 & 245.2 & 142.7 & 97.2 & 146.5 & 109.6 & 9.200 & 5.629 \\
\hline $\mathrm{mtf} 7$ & 1273 & 3.442 & 238.8 & 141.4 & 97.0 & 144.3 & 107.2 & 9.133 & 5.578 \\
\hline$m t f 8^{a}$ & 1073 & 3.474 & 250.7 & 144.6 & 95.8 & 147.4 & 112.6 & 9.253 & 5.691 \\
\hline $\mathrm{mtf} 9$ & 1373 & 3.426 & 232.4 & 139.6 & 96.5 & 141.8 & 104.6 & 9.059 & 5.522 \\
\hline $\mathrm{mtf} 10$ & $1510(10)$ & 3.405 & 224.5 & 136.3 & 97.2 & 139.6 & 100.4 & 8.961 & 5.427 \\
\hline $\mathrm{mtf} 11^{\mathrm{a}}$ & 1073 & 3.474 & 252.0 & 144.8 & 96.9 & 148.6 & 112.7 & 9.275 & 5.694 \\
\hline
\end{tabular}

${ }^{\mathrm{a}}$ Measurements were performed on the temperature decrease.

tures were within $1^{\circ}$. At other temperatures the data were usually collected in six to seven directions since the sample orientation was already known. We wish to point out that the high-temperature cell allows a high density of data points to be collected over a wide range of crystallographic directions in a scattering plane. There is a very low root mean square (RMS) error of the data due to the well controlled scattering geometry (Fig. 9).

Calculation of the elastic moduli from the measured velocities requires knowledge of the density as a function of temperature. The density of $\mathrm{MgO}$ at each temperature was calculated from the thermal expansion data of Suzuki, ${ }^{20}$ measured to $1273 \mathrm{~K}$, and the data of Dubrovinsky and Saxena, ${ }^{21}$ measured to the melting point $(\sim 3100 \mathrm{~K})$. These two data sets are in agreement (less than $0.03 \%$ difference) within our experimental temperature range.

Acoustic velocities were inverted for the elastic moduli using a linearized inversion procedure described by Weidner and Carleton. ${ }^{22}$ The final best-fit elastic constant models yielded $7-19 \mathrm{~m} / \mathrm{s} \mathrm{rms}$ error with respect to the observed velocity data. The $C_{i j}$ 's for $\mathrm{MgO}$, as well as the aggregate elastic moduli [Voigt-Reuss-Hill (VRH) average] and acoustic velocities as a function of temperature are given in Table I. In Figs. 7 and 8 our results are compared with ultrasonic data of Sumino et al. ${ }^{16}$ and Isaak et al. ${ }^{17}$ Our Brillouin results are in agreement with the ultrasonic measurements within the experimental uncertainties. No systematic deviations from the trend of the ultrasonic results were observed.

Our single-crystal elastic constants show essentially linear behavior with temperature, yielding the temperature derivatives of $-0.060(2),-0.015(1)$, and $+0.001(1) \mathrm{GPa} / \mathrm{K}$ for $C_{11}, C_{44}$, and $C_{12}$, respectively. The linear temperature derivatives for the adiabatic bulk and shear moduli are $-0.019(2)$ and $-0.024(2) \mathrm{GPa} / \mathrm{K}$ in the temperature range from 295 to $1510(10) \mathrm{K}$.

The excellent agreement with other experimental data demonstrates that the cell can be effectively and reliably used for high-temperature single-crystal elasticity measurements on very small crystals to temperatures in excess of $1500 \mathrm{~K}$.

\section{ACKNOWLEDGMENTS}

This research was supported by the NSF. One of the authors (J. M. J.) was supported in part by the Research Experiences for Undergraduates program of the NSF. The work of another author (J. W. P.) was supported by a Fannie and John Hertz Foundation graduate fellowship.

${ }^{1}$ E. S. Zouboulis and M. Grimsditch, J. Appl. Phys. 70, 772 (1991).

${ }^{2}$ V. Askapour, M. H. Manghnani, S. Fassbender, and A. Yoneda, Phys. Chem. Miner. 19, 511 (1993).

${ }^{3}$ J. A. Bucaro and H. D. Dardy, J. Appl. Phys. 45, 5324 (1974).

${ }^{4}$ J. Pelous and R. Vacher, Solid State Commun. 18, 657 (1976).

${ }^{5}$ J. E. Masnik, J. Kieffer, and J. D. Bass, J. Am. Ceram. Soc. 76, 3073 (1993).

${ }^{6}$ P. E. Ngoepe and J. D. Comins, Phys. Rev. Lett. 61, 978 (1988).

${ }^{7}$ P. E. Ngoepe and J. D. Comins, J. Less-Common Met. 148, 375 (1989).

${ }^{8}$ P. J. Botha, J. C. H. Chiang, J. D. Comins, P. M. Mjwara, and P. E. Ngoepe, J. Appl. Phys. 73, 7268 (1993).

${ }^{9}$ P. E. Ngoepe and J. D. Comins, J. Phys. C 20, 2983 (1987).

${ }^{10}$ P. R. Stoddart, P. E. Ngoepe, P. M. Mjwara, J. D. Comins, and G. A. Saunders, J. Appl. Phys. 73, 7298 (1993).

${ }^{11}$ C. H. Whitfield, E. M. Brody, and W. A. Bassett, Rev. Sci. Instrum. 47, 942 (1976).

${ }^{12}$ C.-S. Zha, T. S. Duffy, R. T. Downs, H.-K. Mao, and R. J. Hemley, J. Geophys. Res. 101, 17535 (1996).

${ }^{13}$ J. M. Jackson, S. V. Sinogeikin, and J. D. Bass, Am. Mineral. (in press).

${ }^{14}$ L. Merill and W. A. Bassett, Rev. Sci. Instrum. 45, 290 (1974).

${ }^{15}$ W. F. Oliver, C. A. Herbst, S. M. Lindsay, and G. H. Wolf, Rev. Sci. Instrum. 63, 1884 (1992)

${ }^{16}$ Y. Sumino, O. L. Anderson, and I. Suzuki, Phys. Chem. Miner. 9, 38 (1983).

${ }^{17}$ D. G. Isaak, O. L. Anderson, and T. Goto, Phys. Chem. Miner. 16, 704 (1989).

${ }^{18}$ E. S. Zouboulis and M. Grimsditch, J. Geophys. Res. 96, 4167 (1991).

${ }^{19}$ S. V. Sinogeikin, T. Katsura, and J. D. Bass, J. Geophys. Res. 103, 20819 (1998).

${ }^{20}$ I. Suzuki, J. Phys. Earth 23, 145 (1975).

${ }^{21}$ L. S. Dubrovinsky and S. K. Saxena, Phys. Chem. Miner. 24, 547 (1997).

${ }^{22}$ D. J. Weidner and H. R. Carleton, J. Geophys. Res. 82, 1334 (1977). 